\title{
Evaluación positiva de medicamentos: 4.` Trimestre 2012
}

\author{
Puerro Vicente M. ${ }^{1}$, García Luque A. ${ }^{2}$, Prats Olivan P. ${ }^{3}$, González Alfonso M. ${ }^{4}$
}

Sanid. mil. 2013; 69 (1): 43-47; ISSN: 1887-8571

\section{RESUMEN}

Se reseñan los medicamentos ya evaluados por la Agencia Española de Medicamentos y Productos Sanitarios hechos públicos en el último trimestre de 2012, y considerados de mayor interés para el profesional sanitario. Se trata de opiniones técnicas positivas que son previas a la autorización y puesta en el mercado del medicamento.

PALABRAS CLAVE: Aflibercept, Zaltrap, Florbetapir ${ }^{18} \mathrm{~F}$, Amyvid, Insulina Degludec, Tresiba, Insulina Degludec/Insulina Aspartato, Ryzodeg, Lixisenatida, Lyxumia, Loxapina, Adasuve, Mirabegron, Betmiga, Nalmefeno, Selincro, Pegloticasa, Krystexxa, Pertuzumab, Perjeta, Vacuna Meningocócica del serogrupo B (de componentes ADNr adsorbida), Bexsero.

\section{Positive assessment of drugs: $4^{\text {th }}$ quarter 2012}

SUMMARY: The drugs assessed by the Spanish Agency for Medicines and Health Products made public in the last quarter of 2012, and considered of interest to the healthcare professional, are reviewed. These are positive technical reports prior to the authorization and placing on the market of the product.

KEY WORDS: Aflibercept, Zaltrap, Florbetapir ${ }^{18} \mathrm{~F}$, Amyvid, Insulina Degludec, Tresiba, Insulina Degludec/Insulina Aspartato, Ryzodeg, Lixisenatida, Lyxumia, Loxapina, Adasuve, Mirabegron, Betmiga, Nalmefeno, Selincro, Pegloticasa, Krystexxa, Pertuzumab, Perjeta, Vacuna Meningocócica del serogrupo B (de componentes ADNr adsorbida), Bexsero.

\section{AFLIBERCEPT $\left(\text { Zaltrap }^{\circledR}\right)^{1-3}$}

Aflibercept es un agente antineoplásico que actúa como receptor cebo del factor de crecimiento del endotelio vascular (VEGF) bloqueando así la vía biológica VEGF. Esta vía es importante para el suministro sanguíneo a los tumores, aunque tiene también descritas otras funciones biológicas.

Está indicado en combinación con irinotecan/5-fluorouracilo/ ácido folínico (FOLFIRI), para el tratamiento de adultos con carcinoma metastásico de colon o recto resistente o que ha progresado tras un régimen de quimioterapia basado en oxaliplatino.

En los ensayos clínicos aflibercept, ha mostrado que mejora la supervivencia de los pacientes y retrasa la progresión de la enfermedad en comparación con placebo. La eficacia y la seguridad de Aflibercept se evaluó en un estudio clínico aleatorizado de 1.226 pacientes con cáncer colorrectal metastásico cuyos tumores progresaron mientras recibían quimioterapia combinada basada en oxaliplatino, o cuyo cáncer había sido extirpado mediante cirugía, pero había recidivado en el plazo de seis meses después de recibir quimioterapia adyuvante basada en oxaliplatino después de la cirugía. Los participantes recibieron el tratamiento hasta que su tumor progresara o los efectos secundarios se convirtieron en inaceptables.

\footnotetext{
1 Tcol. Médico. Servicio de Farmacología Clínica.

2 Cte. Médico. Servicio de Farmacología Clínica.

3 Cte. Farmacéutico. Servicio de Farmacia Hospitalaria.

${ }^{4}$ Cor. Farmacéutico Servicio de Farmacia Hospitalaria

Hospital Central de la Defensa Gómez Ulla. Madrid. España.
}

Dirección para correspondencia: Servicio de Farmacología Clínica. Hospital Central de la Defensa Gómez Ulla. 28047 Madrid. España. mpuevi1@oc.mde.es

Recibido: 8 de enero de 2013

Aceptado: 29 de enero de 2013
El estudio fue diseñado para medir la supervivencia global. Los pacientes que fueron asignados a recibir la combinación Aflibercept más FOLFIRI vivieron un promedio de 13,5 meses, comparado con un promedio de 12 meses para los que recibieron FOLFIRI más placebo. También se observó una reducción en el tamaño del tumor en el 20 por ciento de los pacientes que recibieron la combinación Aflibercept más FOLFIRI en comparación con 11 por ciento para los que recibieron FOLFIRI más placebo.

Además, el ensayo clínico demostró una mejora en la supervivencia libre de progresión. La supervivencia libre de progresión en pacientes que recibieron la combinación Aflibercept más FOLFIRI fue de 6,9 meses en comparación con 4,7 meses para los que recibieron FOLFIRI más placebo.

En USA Zaltrap ${ }^{\circledR}$ se aprobó con un recuadro de advertencia alertando a los pacientes y los profesionales de la salud de que el fármaco puede causar hemorragia grave y a veces fatal, incluyendo sangrado gastrointestinal, y el desarrollo de perforaciones en el tracto gastrointestinal. Así mismo el fármaco también puede hacer que sea más difícil curar las heridas.

Las reacciones adversas observadas con más frecuencia durante el desarrollo clínico fueron: leucopenia, diarrea, neutropenia, proteinuria, aumento de los niveles de aspartato aminotranferasa, estomatitis, fatiga, trombocitopenia, aumento de los niveles de alanina aminotransferasa, hipertensión, pérdida de peso, disminución del apetito, epistaxis, dolor abdominal, disfonía, aumento de los niveles de creatinina sérica y cefalea.

Se recomienda su prescripción por médicos con experiencia en el tratamiento antineoplásico.

El 15 de noviembre de 2012, el Comité de Medicamentos de Uso Humano de la Agencia Europea de Medicamentos emitió una opinión positiva, recomendando la concesión de una auto- 
rización de comercialización del medicamento Zaltrap ${ }^{\circledR}$, e igualmente fue aprobado por la FDA estadounidense el 3 de agosto de 2012

\section{FLORBETAPIR ${ }^{18}$ F $\left(\text { Amyvid }^{\circledR}\right)^{4-6}$}

Radiofármaco de uso diagnóstico, que debe utilizarse junto con la exploración clínica para la obtención de imágenes, mediante tomografía por emisión de positrones (PET), de la densidad de placa neurítica de $\beta$-amieloide en el cerebro de adultos con deterioro cognitivo que están siendo evaluados por enfermedad de Alzheimer y otras causas de deterioro cognitivo.

Florbetapir $\left({ }^{18} \mathrm{~F}\right)$, se une a las placas neuríticas de $\beta$-amieloide en la sustancia gris del cerebro. Dichas placas pueden encontrarse presentes en ancianos sin deterioro cognitivo y en determinadas demencias neurodegenerativas (enfermedad de Alzheimer, demencia de Parkinson y demencia de cuerpos de Lewy).

En ensayos clínicos realizados en pacientes terminales, florbetapir $\left({ }^{18} \mathrm{~F}\right)$ ha detectado la presencia de placas neuríticas de $\beta$-amieloide de densidad de moderada a frecuente en el cerebro, lo que hasta el momento solo era posible mediante autopsia post-mortem. En estos estudios la correlación demostrada entre las lecturas de varios radiólogos diferentes y los posteriores hallazgos postmorten fueron positivas.

En combinación con el diagnóstico clínico, una imagen PET negativa con florbetapir $\left({ }^{18} \mathrm{~F}\right)$ puede descartar el diagnóstico de enfermedad de Alzheimer. Una imagen PET positiva con florbetapir $\left({ }^{18} \mathrm{~F}\right)$ no establece el diagnóstico de enfermedad de Alzheimer, ya que el acúmulo de placas neuríticas de $\beta$-amieloide puede estar presente en ancianos sin deterioro cognitivo y en determinadas demencias neurodegenerativas.

En el momento actual, y a falta de lograr una mayor experiencia, su utilidad parece muy limitada ya que no existen datos que muestren si la presencia de placas neuríticas de $\beta$-amieloide en pacientes con alteración cognitiva mínima puede predecir el desarrollo de Alzheimer, ni se ha establecido la eficacia del florbetapir $\left({ }^{18} \mathrm{~F}\right)$ para monitorizar la respuesta al tratamiento. También se desconoce si el diagnóstico con florbetapir $\left({ }^{18} \mathrm{~F}\right)$ mejora el manejo y/o el resultado clínico de los pacientes.

Las reacciones adversas observadas con más frecuencia durante el desarrollo clínico fueron cefalea y disgeusias (alteraciones del gusto). Al igual que otros radiofármacos, la administración de este medicamento supone una pequeña cantidad de radiación. También existe un riesgo de falsos resultados en la interpretación de las imágenes PET con florbetapir $\left({ }^{18} \mathrm{~F}\right)$.

La exploración con florbetapir $\left({ }^{18} \mathrm{~F}\right)$ deben estar restringida a la solicitud de médicos con experiencia en el manejo clínico de trastornos neurodegenerativos, y debe interpretarse por personal con experiencia en imágenes PET con florbetapir $\left({ }^{18} \mathrm{~F}\right)$.

\section{INSULINA DEGLUDEC (Tresiba $\left.{ }^{\circledR}\right)^{4,7}$}

El principio activo es insulina degludec, una insulina basal de acción ultralenta. La insulina degludec se une específicamente al receptor humano de insulina produciendo los mismos efectos farmacológicos que la insulina humana.

Su indicación, como la de otras insulinas, es el tratamiento de diabetes mellitus en pacientes adultos. Se ha desarrollado en dos concentraciones diferentes (100 UI/ml y $200 \mathrm{UI} / \mathrm{ml}$ ).

En los ensayos clínicos ha mostrado su capacidad para disminuir la glucemia y presenta un riesgo menor de hipoglucemia nocturna en comparación con insulina glargina.

La reacción adversa observada con más frecuencia durante el desarrollo clínico fue la hipoglucemia.

El medicamento ya ha sido aprobado en Japón en septiembre de 2012.

\section{INSULINA DEGLUDEC/ INSULINA ASPARTATO $\left(\text { Ryzodeg }^{\circledR}\right)^{4,8}$}

Los principios activos son la insulina degludec que es una insulina basal de acción ultralenta, ya comentada anteriormente, y de la insulina aspartato de acción rápida prandial. La insulina degludec y la insulina aspartato se unen específicamente al receptor humano de insulina produciendo los mismos efectos farmacológicos que la insulina humana.

La indicación es el tratamiento de diabetes mellitus en pacientes adultos.

En los ensayos clínicos ha mostrado capacidad de disminuir la concentración sanguínea de HbA1c (hemoglobina glicosilada) y presenta un menor riesgo de hipoglucemia nocturna en comparación con insulina glargina.

La reacción adversa observada con más frecuencia durante el desarrollo clínico fue la hipoglucemia.

\section{LIXISENATIDA $\left(\text { Lyxumia }^{\circledR}\right)^{1,9}$}

Lixisenatida es un medicamento análogo al péptido similar a glucagón-1 (GLP-1). Como el GLP-1 original, lixisenatida estimula la liberación de insulina por los islotes pancreáticos, suprime la secreción de glucagón, retrasa el vaciamiento gástrico y reduce el peso corporal.

Su indicación aprobada es el tratamiento de la diabetes mellitus tipo 2 en pacientes adultos en combinación con otros hipoglucemiantes orales y/o insulina basal cuando éstos, junto con la dieta y el ejercicio, no consiguen un control adecuado de la glucemia.

Dos ensayos clínicos denominados GetGoal Duo 1 y GetGoal-L2 demostraron la variable principal de eficacia de mejora de la HbAlc con la correspondiente reducción significativa en la glucosa postprandial, demostrando así su capacidad para mejorar el metabolismo de la glucosa cuando se añade a otros antidiabéticos orales y/o a insulina.

Las reacciones adversas observadas con más frecuencia durante el desarrollo clínico fueron: náuseas, vómitos y diarrea. Además, se produjeron hipoglucemia (cuando fue administrado en combinación con sulfonilureas y/o insulina basal) y cefaleas con frecuencia. Se han comunicado reacciones alérgicas en el $0,4 \%$ de los pacientes tratados con lixisenatida. 


\section{Evaluación positiva de medicamentos: $4 .^{\circ}$ trimestre 2012}

\section{LOXAPINA $\left(\text { Adasuve }^{\circledR}\right)^{10-12}$}

El principio activo loxapina, es un antipsicótico y psicoléptico, antagonista de alta afinidad de los receptores de dopamina D2 y de serotonina 5-HT2A. También se une a los receptores noradrenérgicos, histaminérgicos y colinérgicos, lo que podría influir en el espectro de sus efectos farmacológicos asociados con efectos tranquilizantes y la supresión de la conducta agresiva.

$\mathrm{Su}$ indicación es el control rápido de la agitación leve o moderada en pacientes adultos con esquizofrenia o trastorno bipolar. Los pacientes deben recibir su tratamiento habitual inmediatamente después de conseguir el control de los síntomas de la agitación aguda.

Esta forma de loxapina se presenta como polvo para inhalación. Loxapina ya se había aprobado en 1975 en USA en su presentación de cápsulas, comprimidos, solución oral y parenteral para administración intramuscular, pero actualmente solo está comercializada la formulación en cápsulas, aunque la discontinuación de la comercialización de los otros formatos no ha tenido razones de seguridad ni de eficacia y parece que han sido puramente comerciales.

Con esta nueva forma de presentación se han llevado a cabo 2 ensayos clínicos de fase 3 doble-ciego, controlados con placebo, que demuestran la eficacia y seguridad de loxapina para el tratamiento de la agitación: un estudio en pacientes con esquizofrenia y otro estudio en pacientes con trastorno bipolar I. En estos ensayos clínicos, se ha observado que loxapina reduce de manera rápida la agitación leve o moderada en estos pacientes. La reducción de la agitación se hizo evidente 10 minutos después de la primera dosis (el primer momento de evaluación), y en las siguientes evaluaciones durante las 24 horas de evaluación. Aproximadamente de un cuarto a casi la mitad de los pacientes, necesitaron de una segunda dosis después de dos horas, para alcanzar un efecto satisfactorio.

Se realizaron también 3 estudios de seguridad pulmonar de Fase I en voluntarios sanos y en pacientes asmáticos o con EPOC. Las reacciones adversas observadas con más frecuencia durante el desarrollo clínico fueron: disgeusia, sedación (incluyendo sedación combinada con somnolencia), fatiga, e irritación de la garganta, si bien aparecieron en porcentajes similares a los que aparecieron con placebo y no se observó ninguna asociación con sedación excesiva. En estudios en pacientes con agitación, se observó como reacción adversa grave pero poco frecuente, el broncoespasmo, mientras que en pacientes con enfermedad de vías aéreas activa, el broncoespasmo se comunicó frecuentemente y a menudo requirió tratamiento con un broncodilatador beta-agonista de corta duración. No se ha observado influencia en los valores de laboratorio ni en el QT/QTc.

Se recomienda su administración en un centro hospitalario bajo la supervisión de un profesional sanitario. Debería disponerse de tratamiento con broncodilatador beta-agonista de corta duración para el tratamiento de posibles efectos secundarios graves respiratorios (broncoespasmo).

\section{MIRABEGRON (Betmiga $\left.{ }^{\circledR}\right)^{4,13,14}$}

Mirabegron es un agonista selectivo del receptor adrenérgico beta 3 humano, predominante en el músculo detrusor. La activación de este receptor adrenérgico beta 3 en el trígono vesical facilita el almacenamiento de la orina mediante el aplanamiento y alargamiento de la base de la vejiga, sin inhibir el vaciamiento vesical, lo que prolonga el tiempo entre las idas al baño para el paciente. El fármaco fue aprobado en 2011 en Japón y en los EE.UU. en junio pasado.

Esta indicado en el tratamiento sintomático de la urgencia y del aumento de la frecuencia miccional, y/o incontinencia de urgencia, que pueden ocurrir en pacientes con síndrome de vejiga hiperactiva.

$\mathrm{Su}$ aprobación europea ha llegado de la mano de tres ensayos clínicos de fase III que enrolaron a 4.622 pacientes, en los que demostró una eficacia superior en comparación con el placebo en el tratamiento de los síntomas de la vejiga hiperactiva, con pacientes que necesitaron ir al lavabo una frecuencia significativamente menor $(\mathrm{p}<0,001)$ y experimentaron menos episodios de incontinencia $(p<0,001)$. Los estudios también mostraron una mejora estadísticamente significativa de la calidad de vida sobre el placebo en términos de satisfacción con el tratamiento y "molestia síntoma».

Las reacciones adversas observadas con más frecuencia durante el desarrollo clínico fueron, a la dosis propuesta de $50 \mathrm{mg}$, un modesto incremento del pulso y de la presión arterial (1 lpm $\mathrm{y} \leq 1 \mathrm{~mm} \mathrm{Hg}$ en comparación con el placebo).

\section{NALMEFENO (Selincro $\left.^{\circledR}\right)^{10,15,16}$}

El principio activo es nalmefeno, un modulador del sistema opioide con un perfil concreto de interacción con los receptores $\mu, \delta$ у $\kappa$. Se trata de un ligando selectivo de los receptores opioides con actividad antagonista sobre los receptores $\mu$ y $\delta$ y parcialmente agonista sobre el receptor $\kappa$. El consumo agudo de alcohol tiene como resultado una liberación de dopamina en la región mesolímbica (facilitado por la liberación de $\beta$-endorfinas), lo que puede provocar refuerzo positivo. Nalmefeno inhibe la vía de recompensa en el cerebro por lo que contrarresta los efectos del refuerzo positivo y por tanto reduce el consumo de alcohol, posiblemente por modulación de estas funciones cortico-mesolímbicas. Nalmefeno se dirige a un nuevo principio en el tratamiento de la dependencia del alcohol mediante la eliminación de deseo de una persona para beber.

Su indicación es la reducción del consumo de alcohol en pacientes adultos con dependencia alcohólica con un nivel de consumo de alto riesgo (superior a $60 \mathrm{~g}$ de alcohol al día en varones y superior a $40 \mathrm{~g}$ de alcohol al día en mujeres), que no tengan síntomas físicos de abstinencia y que no requieran desintoxicación inmediata.

En ensayos clínicos, nalmefeno ha mostrado su capacidad para reducir el consumo de alcohol en pacientes adultos con dependencia al alcohol con un nivel de consumo de alto riesgo. Se han llevado a cabo tres estudios de fase 2 y dos estudios de Fase 3 en la dependencia del alcohol con nalmefeno. En el más grande de los últimos estudios de Fase 3, se investigaron 400 pacientes alcohólicos en Europa, y demostró la capacidad de nalmefeno de limitar de manera significativa tanto la ingesta de alcohol del paciente como el número promedio de días de consumo excesivo de alcohol (consumo de más de 5 bebidas estándar de alcohol). Se realizaron otros tres estudios clínicos de fase 3 en Europa en 2008 con la participación de 1.997 pacientes. El programa se completó en 2011, y los resultados de la Fase 3 del programa se presentaron en marzo de 2012. Todos los estudios fueron multi- 
céntricos, aleatorizados, doble ciego, controlados con placebo, con grupos paralelos, con dosis de $18 \mathrm{mg}$ tomados a demanda, en pacientes con dependencia al alcohol según el DSM-IV. Los pacientes fueron instruidos para tomar un comprimido recubierto en cada día cuando se percibe un riesgo de consumo de alcohol, preferiblemente una a dos horas antes de la hora prevista de beber.

Las reacciones adversas observadas con más frecuencia durante el desarrollo clínico fueron bastante inespecíficas, como náuseas y mareos. Algunos pacientes comunicaron también la aparición de trastornos del sueño (tanto de día como de noche), que fueron leves y transitorios. La mayoría de estos eventos aparecieron en los primeros días después de la dosis inicial, para luego disminuir con la continuación del tratamiento

Se recomienda su prescripción junto con apoyo psicosocial continuo dirigido a la adherencia al tratamiento y a la reducción del consumo de alcohol. Sólo se debería iniciar el tratamiento con nalmefeno en pacientes que siguen teniendo un nivel de consumo de alto riesgo dos semanas después de la evaluación inicial.

\section{PEGLOTICASA (Krystex $\left.x a^{\circledR}\right)^{4,17,18}$}

El principio activo es pegloticasa, una uricasa recombinante de mamífero pegilada mediante unión covalente con polietilenglicol, de la clase terapéutica de agentes biouricolíticos que disminuyen el ácido úrico en suero.

Tratamiento de la gota crónica tofácea grave debilitante en pacientes adultos que pueden presentar también afectación erosiva de las articulaciones y en quienes no se han podido normalizar los niveles de ácido úrico sérico con inhibidores de la xantina oxidasa a las dosis máximas recomendadas o en aquellos en los que estos medicamentos están contraindicados. No está indicado para el tratamiento de la hiperuricemia asintomática. La decisión de tratar con pegloticasa se debe basar en una evaluación continua del balance beneficio-riesgo individualizado.

En ensayos clínicos pegloticasa ha mostrado capacidad para reducir el ácido úrico sérico a niveles indetectables en pacientes que han fracasado con tratamientos convencionales (inhibidores de la xantina oxidasa o agentes uricosúricos). En ensayos Fase III se estudió la eficacia y seguridad del fármaco con la administración de $8 \mathrm{mg}$ por infusión intravenosa cada 2 semanas. Pegloticasa consiguió que el objetivo de reducir el ácido úrico por debajo de los $6 \mathrm{mg} / \mathrm{dl}$ fuese conseguido por al menos el $80 \%$ del tiempo durante 3 y 6 meses comparado con el tratamiento con placebo. El 47\% (20/43) y el 38\% (16/42) de los pacientes con pegloticasa a las dosis descritas consiguieron el objetivo primario mientras que en los pacientes con placebo el resultado fue del $0 \%$ $(0 / 20$ y $0 / 23$, respectivamente) $(\mathrm{p}<0,001)$. La resolución de los tofos a los 6 meses fue completa en el 45\% (18/40) de los pacientes con pegloticasa vs $8 \%(2 / 25)$ con placebo $(\mathrm{p}=0,002)$.

No está exento de efectos adversos que a veces pueden ser graves. Las reacciones adversas más frecuentes fueron las crisis de gota $(77 \%)$, reacciones de infusión $(26 \%)$, náuseas $(12 \%)$, contusión o equimosis $(11 \%)$, nasofaringitis $(7 \%)$, estreñimiento $(6 \%)$, dolor de pecho $(6 \%)$, anafilaxia $(5 \%)$ y vómitos $(5 \%)$. Además de estos eventos que se producen en más del $5 \%$, también ha aparecido exacerbación de la insuficiencia cardíaca congestiva preexistente, en un $2 \%$.

Se recomienda que el tratamiento con pegloticasa sea iniciado y supervisado por médicos con experiencia en el diagnóstico y tratamiento de la gota crónica refractaria grave. Se debe administrar en centros sanitarios y por profesional sanitario con experiencia en el manejo de la anafilaxia y de reacciones debidas a la infusión intravenosa. Se debe monitorizar al paciente durante la infusión intravenosa y al menos durante la primera hora tras el final de la infusión. Debe disponerse de un equipo de reanimación.

\section{PERTUZUMAB $\left(\text { Perjeta }^{\circledR}\right)^{10,19-21}$}

Pertuzumab es un anticuerpo monoclonal recombinante humanizado específico del dominio de dimerización extracelular (subdominio II) del receptor 2 del factor de crecimiento epidérmico humano (HER2) y, debido a esto, bloquea la heterodimerización dependiente de ligando de HER2 con otros miembros de la familia HER, que incluyen EGFR (receptor del factor de crecimiento epidérmico), HER3 y HER4. Pertuzumab inhibe la señalización intracelular iniciada mediante ligando a través de dos vías de señalización principales: proteína quinasa activada por mitógeno (MAP) y fosfoinositol 3-quinasa (PI3K). La inhibición de estas vías de señalización puede producir una detención del crecimiento celular y apoptosis, respectivamente. Además, pertuzumab es un mediador de la citotoxicidad celular dependiente de anticuerpos (ADCC).

Pertuzumab se utiliza en combinación con trastuzumab y docetaxel en pacientes adultos con cáncer de mama irresecable localmente recurrente o metastático, HER2 positivo, que no han recibido previamente tratamiento anti-HER2 o quimioterapia para su enfermedad metastásica. La detección de la sobreexpresión de la proteína HER2 es necesaria para la selección de pacientes adecuados para ser tratados con pertuzumab ya que son los únicos pacientes estudiados y para quienes se ha demostrado beneficio.

En ensayos clínicos, pertuzumab ha mostrado tener capacidad de mejorar la supervivencia libre de progresión, la supervivencia global y la tasa de respuesta objetiva en pacientes tratados con pertuzumab en comparación con placebo. Hasta ahora el fármaco se ha estudiado en más de 1.400 pacientes con diferentes enfermedades y casi siempre en combinación con otros fármacos. En el principal ensayo clínico multicéntrico, doble ciego, y aleatorizado (CLEOPATRA), intervinieron 806 pacientes procedentes de Europa, Norteamérica, Sudamérica y Asia. El estudio puso en evidencia un incremento en el intervalo libre de progresión de 6,1 meses (media de 18,5 meses en el grupo tratado con pertuzumab vs 12,4 meses en el grupo tratado con placebo).

Las reacciones adversas más comunes (>30\%) que se observaron con pertuzumab en combinación con trastuzumab y docetaxel fueron diarrea, alopecia, neutropenia, náuseas, fatiga, erupciones cutáneas y neuropatía periférica. Las reacciones adversas más comunes con Grado 3-4 (>2\%) fueron neutropenia, neutropenia febril, leucopenia, diarrea, neuropatía periférica, anemia, astenia y fatiga. Un aumento en la incidencia de neutropenia febril se observó en los pacientes asiáticos en comparación con los pacientes de otras razas y de otras regiones geográficas. Entre los pacientes asiáticos, la incidencia de neutropenia febril 
fue mayor en el grupo tratado con pertuzumab (26\%) en comparación con el grupo tratado con placebo (12\%). La exposición a pertuzumab puede causar daño fetal cuando se administra a una mujer embarazada y puede resultar en muerte embriofetal y defectos de nacimiento. Los estudios en animales han dado lugar a oligohidramnios, retraso en el desarrollo renal y muerte.

Se recomienda su prescripción por médicos con experiencia en el tratamiento antineoplásico.

\section{VACUNA MENINGOCÓCICA DEL SEROGRUPO B (DE COMPONENTES ADNR, ADSORBIDA) $\left(\text { Bexsero }^{\circledR}\right)^{1,22}$}

Se trata de una vacuna meningocócica del serogrupo B (de componentes $\mathrm{ADNr}$, adsorbida) que estimula la producción de anticuerpos antibacterianos contra los antígenos de la vacuna (NHBA, NadA, fHbp y PorA P1.4).

El 15-11-2012 el Comité de Medicamentos de Uso Humano (CHMP) de la Agencia Europea de Medicamentos (EMA) adoptó una opinión positiva para Bexsero ${ }^{\circledR}$.

Está indicado para la inmunización activa de niños desde los 2 meses de edad, adolescentes y adultos para la prevención de la enfermedad invasiva meningocócica causada por Neisseria meningitidis del serogrupo B.

Actualmente las vacunas disponibles no ofrecen una protección amplia contra el meningococo del serogrupo B, que representa hasta un $90 \%$ de todos los casos de enfermedad meningocócica en algunos países europeos. Esta infección es fácilmente mal diagnosticada, pudiendo causar la muerte en un plazo de 24 horas $\mathrm{u}$ ocasionar graves discapacidades de por vida. Alrededor de 1 de cada 10 de las personas que contraen esta enfermedad mueren a pesar del tratamiento apropiado. Hasta una de cada cinco supervivientes sufre discapacidades devastadoras para toda la vida tales como daño cerebral, pérdida de audición o pérdida de la integridad física. Las tasas más altas de enfermedad ocurren en el primer año de vida, alcanzando un máximo a los 7 meses de edad.

El uso de la vacuna deberá determinarse en base a las recomendaciones oficiales. El impacto de la enfermedad invasiva en diferentes grupos de edad, así como la variabilidad de la epidemiología de los antígenos de las cepas del serogrupo B en diferentes áreas geográficas.

El perfil de tolerabilidad e inmunogenicidad de Bexsero ${ }^{\circledR}$ se ha establecido a través de un amplio programa clínico, e incluyó datos de grandes ensayos clínicos Fase II/III que incluyeron a casi 8.000 bebés, niños, adolescentes y adultos. En ensayos clínicos, la vacuna meningocócica del serogrupo $\mathrm{B}$, ha mostrado que protege contra la enfermedad invasiva meningocócica causada por Neisseria meningitidis del serogrupo B.

Las reacciones adversas observadas con más frecuencia durante el desarrollo clínico fueron: fiebre, somnolencia, diarrea, vómitos, rash cutáneo, dolor en el lugar de la inyección, mialgia y artralgia.

\section{BIBLIOGRAFÍA}

1. Agencia Española de Medicamentos y Productos Sanitarios. Informe mensual sobre Medicamentos de Uso Humano y Productos Sanitarios Noviembre 2012. Disponible en: http://www.aemps.gob.es/informa/informeMensual/2012/noviembre/home.htm
2. Committee for Medicinal Products for Human Use (CHMP). Zaltrap. Disponible en: http://www.ema.europa.eu/ema/index.jsp?curl=pages/medicines/ human/medicines/002532/smops/Positive/human_smop_000445.jsp\&mid=W C0b01ac058001d127\&source $=$ homeMedSearch\&category=human

3. Food and Drugs Administration. Zaltrap. Disponible en: http://www.fda gov/NewsEvents/Newsroom/PressAnnouncements/ucm314372.htm

4. Agencia Española de Medicamentos y Productos Sanitarios. Informe mensual sobre Medicamentos de Uso Humano y Productos Sanitarios Octubre 2012. Disponible en: http://www.aemps.gob.es/informa/informeMensual/2012/octubre/home.htm

5. Committee for Medicinal Products for Human Use (CHMP). Amyvid. Disponible en: http://www.ema.europa.eu/ema/index.jsp?curl=pages/news_and_ events/news/2012/10/news_detail_001639.jsp\&mid=WC0b01ac058004d5cl

6. Food and Drugs Administration. Amyvid. Disponible en: http://www.fda.gov/ downloads/AdvisoryCommittees/CommitteesMeetingMaterials/Drugs/PeripheralandCentralNervousSystemDrugsAdvisoryCommittee/UCM240951.pdf

7. Committee for Medicinal Products for Human Use (CHMP). Tresiba. Disponible en: http://www.ema.europa.eu/ema/index.jsp?curl=pages/medicines/ human/medicines/002498/smops/Positive/human_smop_000437.jsp\&mid=W C0b01ac058001d127\&source $=$ homeMedSearch\&category $=$ human

8. Committee for Medicinal Products for Human Use (CHMP). Ryzodeg. Disponible en: http://www.ema.europa.eu/ema/index.jsp?curl=pages/medicines/ human/medicines/002499/smops/Positive/human_smop_000436.jsp\&mid=W C0b01ac058001d127\&source $=$ homeMedSearch\&category $=$ human

9. Committee for Medicinal Products for Human Use (CHMP). Lyxumia. Disponible en: http://www.ema.europa.eu/ema/index.jsp?curl=pages/medicines/ human/medicines/002445/smops/Positive/human_smop_000448.jsp\&mid=W C0b01ac058001d127\&source $=$ homeMedSearch\&category $=$ human

10. Agencia Española de Medicamentos y Productos Sanitarios. Informe mensual sobre Medicamentos de Uso Humano y Productos Sanitarios Diciembre 2012. Disponible en: http://www.aemps.gob.es/informa/informeMensual/2012/diciembre/home.htm

11. Committee for Medicinal Products for Human Use (CHMP). Adasuve. Disponible en: http://www.ema.europa.eu/ema/index.jsp?curl=pages/medicines/ human/medicines/002400/smops/Positive/human_smop_000456.jsp\&mid=W C0b01ac058001d127\&source $=$ homeMedSearch\&category $=$ human

12. Food and Drugs Administration. Adasuve. Disponible en: http://www.fda. gov/downloads/AdvisoryCommittees/CommitteesMeetingMaterials/Drugs/ PsychopharmacologicDrugsAdvisoryCommittee/UCM282900.pdf

13. Committee for Medicinal Products for Human Use (CHMP). Betmiga. Disponible en: http://www.ema.europa.eu/ema/index.jsp?curl=pages/medicines/ human/medicines/002388/smops/Positive/human_smop_000433.jsp\&mid=W C0b01ac058001d127\&source $=$ homeMedSearch\&category $=$ human

14. Food and Drugs Administration. Mirabegron. Disponible en: http://www. fda.gov/downloads/AdvisoryCommittees/CommitteesMeetingMaterials/ Drugs/ReproductiveHealthDrugsAdvisoryCommittee/UCM298284.pdf

15. Committee for Medicinal Products for Human Use (CHMP). Selincro. Disponible en: http://www.ema.europa.eu/ema/index.jsp?curl=pages/medicines/ human/medicines/002583/smops/Positive/human_smop_000458.jsp\&mid=W C0b01ac058001d127\&source $=$ homeMedSearch\&category $=$ human

16. Biotie. Selincro Disponible en: http://www.biotie.com/en/product_and_development/central_nervous_system_disorders/selincro

17. Committee for Medicinal Products for Human Use (CHMP). Krystexxa. Disponible en: http://www.ema.europa.eu/ema/index.jsp?curl=pages/medicines/human/medicines/002208/smops/Positive/human_smop_000405.jsp\&mi $\mathrm{d}=$ WC0b01ac058001d127\&source=homeMedSearch\&category=human

18. Savient. Krystexxa. Disponible en: http://www.krystexxa.com/hcp/efficacy.aspx

19. Committee for Medicinal Products for Human Use (CHMP). Perjeta. Disponible en: http://www.ema.europa.eu/ema/index.jsp?curl=pages/medicines/ human/medicines/002547/smops/Positive/human_smop_000457.jsp\&mid=W C0b01ac058001d127\&source $=$ homeMedSearch\&category $=$ human

20. Foods and drugs administration (FDA). Perjeta. Highlights of prescribing information. Disponible en: http://www.accessdata.fda.gov/drugsatfda_docs/ label/2012/1254091bl.pdf

21. Genentech. Perjeta. Disponible en: http://www.perjeta.com/pdf/perjeta-coredata-brochure.pdf

22. Committee for Medicinal Products for Human Use (CHMP). Bexsero. Disponible en: http://www.ema.europa.eu/ema/index.jsp?curl=pages/medicines/ human/medicines/002333/smops/Positive/human_smop_000447.jsp\&mid=W C0b01ac058001d127\&source $=$ homeMedSearch\&category=human 